\title{
Note sulla rappresentazione letteraria della Vergine nella Commedia
}

Notes sur la représentation littéraire de la Vierge dans la Divine Comédie

Notes on the Literary Representation of the Virgin in the Divine Comedy

\section{Stefano Prandi}

\section{(2) OpenEdition}

\section{Journals}

\section{Edizione digitale}

URL: https://journals.openedition.org/cei/9820

DOI: $10.4000 /$ cei.9820

ISSN: 2260-779X

\section{Editore}

UGA Éditions/Université Grenoble Alpes

\section{Edizione cartacea}

ISBN: 978-2-37747-304-5

ISSN: $1770-9571$

\section{Notizia bibliografica digitale}

Stefano Prandi, «Note sulla rappresentazione letteraria della Vergine nella Commedia», Cahiers d'études italiennes [Online], 33 | 2021, online dal 01 octobre 2021, consultato il 13 octobre 2021. URL: http:// journals.openedition.org/cei/9820 ; DOl: https://doi.org/10.4000/cei.9820

Questo documento è stato generato automaticamente il 13 octobre 2021 


\title{
Note sulla rappresentazione letteraria della Vergine nella commedia
}

\author{
Notes sur la représentation littéraire de la Vierge dans la Divine Comédie \\ Notes on the Literary Representation of the Virgin in the Divine Comedy
}

\author{
Stefano Prandi
}

1 L'argomento che qui si propone non è privo di insidie e parrà, a seconda dei punti di vista, da un lato troppo pretenzioso e dall'altro troppo angusto. Chi scrive è convinto che la Commedia abbia rappresentato per il suo autore soprattutto una sfida ai limiti della finzione letteraria: sfida audacissima, certo, in cui ad essere coinvolto è l'uomo nella totalità della propria dimensione spirituale, dunque anche nel sentimento religioso, ma pur sempre inassimilabile alla pura intenzione speculativa e, meno che mai, al semplice abbandono devozionale. Sta di fatto, però, che il poema si confronta continuamente con argomenti di carattere dogmatico, e ciò appare particolarmente vero nel caso della Vergine Maria, oggetto di una serie di controversie all'epoca di Dante - si pensi all'Immacolata concezione - che avrebbero potuto forse opporre resistenza al lavoro dell'«alta fantasia». A ciò si aggiunge, nel caso specifico, un fattore apparentemente favorevole ma in realtà foriero di pericolosa entropia, come l'enorme e ramificato sviluppo tematico dei motivi mariani derivati dalla tradizione leggendaria, liturgica, innografica, confluiti poi in repertori come lo Speculum Beatae Mariae Virginis di Corrado di Sassonia o il Mariale aureum. Un "rumore di fondo" che ha inevitabilmente raggiunto la letteratura critica, incline spesso a sopravvalutare talune di queste pretese "fonti" nonostante il carattere pervasivo delle analogie riscontrate. Rappresentare la figura della Vergine in un'opera dalla struttura tanto complessa e dalla significazione simbolica così stratificata quale la Commedia ha dunque costituito, per l'autore, un cimento di straordinario impegno. Un problema prima di tutto letterario: Maria, dall'inizio alla fine del poema, non smarrisce mai il proprio carattere di «poetic artefact $»^{1}$. 
2 Le strategie individuate da Dante per far fronte a un compito tanto arduo mi paiono sostanzialmente due. Innanzitutto il ricorso a un'animazione progressiva della figura della Vergine, non soltanto da una cantica all'altra ma anche all'interno delle ultime due; in secondo luogo la costante fedeltà a una rigorosa economia di scelte tematiche ed espressive rispetto all'esuberanza di materiali narrativi e di forme che coinvolgono la letteratura mariana, in una sintesi che finisce per esaltare l'originalità della scrittura dantesca. Esporrò dunque le mie brevi considerazioni su entrambi i punti, seguendo la scansione narrativa del poema.

\section{Il prologo infernale}

3 La prima epifania mariana è posta come quinta dell'intera avventura oltremondana del protagonista:

Donna è gentil nel ciel che si compiange

di questo 'mpedimento ov'io ti mando,

sì che duro giudicio là sù frange.

Questa chiese Lucia in suo dimando

e disse: - Or ha bisogno il tuo fedele

di te, e io a te lo raccomando -.

(Inf. II, 94-99)

4 Come ricorda Hollander nel suo commento, nessuno dei commentatori antichi, almeno

fino a Castelvetro, identifica la «donna gentil» con Maria, preferendo far riferimento più genericamente alla "profonda mente della deità» (Jacopo Alighieri), o alla divina grazia che anticipa le decisioni dell'uomo preparandolo alle virtù (Pietro Alighieri, Benvenuto da Imola, Ottimo, Francesco da Buti). Che la triade completata poi dall'intervento di santa Lucia e Beatrice possa comportare l'assenza della figura ausiliatrice per eccellenza mi pare da escludere ${ }^{2}$. Si tratta, comunque, dell'unico riferimento alla Vergine di tutta la prima cantica, peraltro allusivo: né questo stupisce, dal momento che anche Cristo non è mai nominato direttamente ma sempre attraverso perifrasi («somma sapienza», Inf. III, 6; «un possente», IV, 53; «nimica podesta», VI, 96; «[...] colui che la gran preda / levò a Dite [...]», XII, 38-39; «un uom», XXIII, 117; «[...] l'uom che nacque e visse sanza pecca», XXXIV, 115) ${ }^{3}$.

5 L'autore assegna dunque a Maria un'entrata in scena assai discreta e quasi in sordina. A questa caratteristica parrebbe conformarsi, all'interno di questo prologo infernale, la dulcedo vitanoviana che, come ha notato la maggior parte degli interpreti, attraversa l'intero racconto di Virgilio a partire dalla menzione di Beatrice ai vv. $52 \mathrm{sgg}$. A questo proposito va notato che l'attacco «Donna è gentil» da un lato allude a un contesto squisitamente lirico - cioè profano-, e dall'altro se ne distacca parzialmente attraverso l'inversione del tradizionale ordo verborum: sono i sintagmi «gentil mia donna» o, meno frequentemente, "gentil donna» che si ritrovano infatti, nella quasi totalità dei casi, in Guittone, Monte Andrea, Chiaro Davanzati, Dante da Maiano e Dante stesso, nella stanza di canzone Lo meo servente core, v. 14 e nel sonetto Ne le man vostre, gentil donna mia. Eccezione significativa, il passaggio tra il primo e il secondo cominciamento del sonetto Era venuta ne la mente mia della Vita nuova ${ }^{4}$ :

Era venuta ne la mente mia

la gentil donna che per suo valore

fu posta da l'altissimo Signore

nel ciel de l'umiltate, ov'è Maria. 
Era venuta ne la mente mia

quella donna gentil cui piange Amore.

Entro 'n quel punto che lo suo valore

vi trasse a riguardar quel ch'eo facia.

[...]

Visti in controluce rispetto a quanto si è detto sinora, i due cominciamenti suggeriscono alcune considerazioni non prive di interesse. Il primo sembra anticipare, nella tensione oppositiva tra "altissimo» e «umiltate» la terzina incipitaria della preghiera alla Vergine di Bernardo (Par. XXXIII, 2, «umile e alta più che creatura»). Il secondo, oltre a presentare l'avvenuta inversione del nesso aggettivale-sostantivale, precorre l'azione del piangere di Inf. II (v. 94: «si compiange»), seppur riferita a Beatrice, e ambienta in uno scenario interiore quel guardare che nel prologo infernale è contemplazione dall'alto delle sfere celesti (santa Lucia a Beatrice: «Non odi tu la pieta del suo pianto, / non vedi tu la morte che 'l combatte / su la fiumana ove 'l mar non ha vanto?», vv. 106-108).

Il ricorso di Inf. II, 96 ad un sintagma verbale eccezionalmente esplicito come frangere, che introduce un'intensificazione semantica proprio nel delicato passaggio che tocca il problema della predestinazione e dell'equilibrio tra immutabilità del giudizio divino $\mathrm{e}$ libero arbitrio umano, segnala poi, a mio giudizio, una spia della forza nascosta nella quieta e silente disponibilità all'ascolto della Vergine. Se è stata evocata in proposito l'influenza del sapienziale «lingua mollis confringet duritiam» (Prov. 25, 15) $)^{5}$, ulteriori riscontri possono essere individuati in ambito lirico, come nel sonetto del Notaro Si alta amanza à pres'a lo me' core. Si tratta di un componimento atipico, perché incentrato non su un tono precettistico ma sull'espressione di una condizione interiore dell'amante, che ha avuto l'ardire di porre il proprio desiderio su una donna irraggiungibile, paragonata infatti ad un'aquila (v.3). Nonostante questo, la sfida è sostenuta con «orgoglio», confidando nella forza del proprio sentimento amoroso: «[...] lo diamante rompe a tutte l'ore / de lacrime lo molle sentimento. // Donqua, madonna, se lacrime e pianto / de lo diamante frange le durezze / vostre altezze potria isbasare» (vv. 7-11)6.

Se il nome di Maria appare impronunciabile all'inferno, è perché esso agisce ben più che montalianamente, come testimonierà il racconto di Guido da Montefeltro che, prendendo congedo dalla vita «nel nome di Maria» (Purg. V,101), si sottrae alla dannazione eterna. Il poeta stesso si affiderà ad esso in una delle rare rievocazioni autobiografiche della Commedia, menzionando la consuetudine assidua della preghiera alla Vergine che lo accompagnerà negli anni difficili del suo esilio: «il nome del bel fior ch'io sempre invoco / e mane e sera» (Par. XXIII, 88-89). Non potrà allora essere casuale che, nel momento cruciale del passaggio attraverso il muro di fuoco purgatoriale, sia appunto il nome di Beatrice ${ }^{7}$ che, adombrando una tipica prerogativa mariana, riesce a smuovere il protagonista, pietrificato dalla «temenza» (v. 31):

Come al nome di Tisbe aperse il ciglio

Piramo in su la morte, e riguardolla,

allor che 'l gelso diventò vermiglio;

così, la mia durezza fatta solla,

mi volsi al savio duca, udendo il nome

che ne la mente sempre mi rampolla.

(Purg. XXVII, 37-42) 


\section{In cammino con Maria}

9 La tradizione critica ha da tempo indicato come quadro contestuale della presenza mariana nel Purgatorio l'esperienza terrena, storica, della Vergine. La vita di Maria rappresenta infatti in modo esemplare le virtù contrapposte ai sette peccati capitali che determinano la suddivisione delle anime in cammino nelle cornici del monte purgatoriale, offrendosi come perfetto modello penitenziale per la loro definitiva purificazione dal peccato. Uno schema desunto in parte nella rassegna delle Beatitudini del Discorso della Montagna, poi codificato già a partire dal XII sec. da autori quali Riccardo di San Vittore ${ }^{8}$. Questa prerogativa fondamentale trova posto all'interno della dimensione liturgica caratteristica della seconda cantica, a partire dal canto dell'antifona Salve Regina, intonata dalle anime nella valletta dei principi negligenti in Purg. VIII. Sulla scansione degli episodi mariani del Purgatorio occorre sottolineare che, pur di fronte alla "plurisecolare assenza di un confine netto e definito tra libri canonici e libri apocrifi ${ }^{9}$, la Commedia sceglie di attenersi rigorosamente al dettato evangelico, senza concedere nulla a tradizioni scritturali come il Protovangelo di Giacomo, dello Pseudo-Matteo e l'Evangelium de nativitate Mariae, da cui pure erano state tratte notizie fondamentali e pienamente condivise nel Medioevo e dallo stesso Dante, come quelle relative ai genitori di Maria, Anna e Gioacchino, e alla sua infanzia, e che avrebbe offerto esempi di virtù in grado di arricchire l'esiguo repertorio presente nei testi canonici. La seconda cantica, più precisamente, esclude qualsiasi accenno alle vicende terrene della Vergine che non riguardano direttamente Cristo, non solo per il periodo precedente alla nascita di quest'ultimo, ma anche per quello successivo alla sua morte. In tutta la Commedia troviamo un solo riferimento, peraltro cursorio, all'Assunzione: un contesto, peraltro, in cui Dante mette in scena in veste di agens ${ }^{10}$ una rettifica nei confronti delle tradizioni leggendarie, nel caso specifico quella che voleva anche l'evangelista Giovanni, al pari di Cristo e Maria, asceso al cielo prima della propria morte $^{11}$. Un episodio che in realtà, attraverso l'accecamento del viator, segnala la sua condizione paradossale di creatura mortale ancora esposta all'errore e bisognosa di speranza da un lato, e dall'altro detentore dell'eccezionale privilegio di trovarsi nel regno dei beati ancora in carne e ossa ${ }^{12}$.

10 La scrupolosa sobrietà della rappresentazione di Maria nel Purgatorio si esercita anche nei confronti della monumentale esegesi patristica e della letteratura devozionale fiorita a partire dagli episodi evangelici. A questo proposito credo sia senz'altro da ridimensionare l'influenza di testi come lo Speculum Beatae Mariae Virginis di Corrado di Sassonia, che alcuni studiosi hanno considerato il modello, se non addirittura la fonte, della rassegna mariana del Purgatorio ${ }^{13}$. Nello Speculum non sono gli episodi evangelici a rappresentare la rassegna esemplare delle virtù della Vergine, poiché il fil rouge argomentativo è costituito piuttosto dal ricorso all'esegesi dei Padri (Bernardo, Anselmo, Agostino, Beda). Manca inoltre una precisa corrispondenza col testo dantesco, dal momento che all'avaritia non è contrapposta la povertà bensì la «liberalitas», intesa come largitas, virtù che consente a Maria di essere inesauribile fonte di bene ${ }^{14}$.

11 Andrà notato piuttosto come la distribuzione dei sette episodi mariani del Purgatorio si presenti secondo una struttura chiastica a doppia cornice che si apre e si chiude con l'evento più decisivo, l'Annunciazione, l'unico che infatti viene ripreso nella mariofania di Par. XXXII, 94-96 («e quello amor che primo lì discese, / cantando 'Ave, Maria, gratia plena', / dinanzi a lei le sue ali distese») ${ }^{15}$. 
Umiltà / superbia: Purg. X, 34-45 - Annunciazione

Carità / invidia: Purg. XIII, 29 - Nozze di Cana

Mansuetudine / ira: Purg. XV, 88-92 - Cristo tra i dottori del tempio

Sollecitudine / accidia: Purg. XVIII, 100 - Visitazione

Povertà / avarizia e prodigalità: Purg. XX, 19-24 - Natività

Sobrietà / gola: Purg. XXII, 142-144 - Nozze di Cana

Castità / lussuria: Purg. XXV, 128 - Annunciazione presenza nei cieli ${ }^{17}$. Ma la citazione evangelica nella processione allegorica dei canti finali del Purgatorio allude anche a un altro fondamentale valore della figura della Vergine, che non è soltanto Mater Christi ma anche Mater Ecclesiae: quella Chiesa trionfante che appunto si mostrerà a Dante nel Paradiso. L'ultimo riferimento alla Vergine del Purgatorio è costituito da un esplicito parallelismo tra Maria e Beatrice, che richiama, nella sobrietà di una semplice formulazione consecutiva, l'episodio del Planctus: «Deus, venerunt gentes', alternando / or tre or quattro dolce salmodia, / le donne incominciaro, e lagrimando; // e Beatrice sospirosa e pia ${ }^{18}$, / quelle ascoltava sì fatta, che poco / più a la croce si cambiò Maria» (XXXIII, 1-6). È a partire da tale doloroso preludio che si prepara il trionfo delle mariofanie degli ultimi canti del Paradiso.

\section{La diritta via dello sguardo}

15 La prima apparizione di Maria nella terza cantica è indiretta, e si manifesta, quasi in dissolvenza, attraverso la preghiera di Piccarda Donati («Così parlommi, e poi cominciò 'Ave, / Maria' cantando, e cantando vanio / come per acqua cupa cosa grave», Par. III, 121-123). Nel nome della Vergine, invocata dalle partorienti (Purg. XX, 19-21), Cacciaguida entra a far parte poi del «dolce ostello» della Firenze antica («Maria mi diè, chiamata in alte grida», Par. XV, 133): uno spunto che si lega a una costellazione di riferimenti all'incarnazione e al parto nella terza cantica ${ }^{19}$, dalla quale comunque non è possibile ricavare alcuna indicazione rispetto alla posizione dantesca sul tema 
controverso dell'immacolata concezione ${ }^{20}$. Al di là di questi riferimenti rapsodici, la presenza mariana all'interno della terza cantica si concentra in due momenti distinti: una sorta di sorprendente prologo, nel canto XXIII, in cui tutti i beati, Cristo stesso e la Vergine si manifestano a Dante nel Cielo delle Stelle fisse, dunque al di fuori, per così dire, del loro luogo naturale, l'Empireo; e, secondariamente, nella sequenza dei canti XXXI-XXXIII ${ }^{21}$.

Il canto XXIII rappresenta una sorta di eccezionale anticipazione della visione finale, e un esempio paradigmatico di come concetti teologici e impulso mistico-devozionale trovino nella Commedia una geniale trasposizione letteraria grazie a una profonda risemantizzazione che conduce da un ambito profano a uno sacro. Il tessuto connettivo del canto, che di fatto consiste in "una successione di visioni mistiche» ${ }^{22}$, è infatti costituito da una serie di transumptiones codificate dalle poetriae medievali - in particolare da Geoffroi de Vinsauf - che insistono su alcune declinazioni del topos del locus amoenus, come la similitudine iniziale dell'«augello» (vv.1-9), quella del plenilunio e del "riso" delle stelle (vv. 25-27), quella infine del giardino paradisiaco in fiore (vv. 70-72) $)^{23}$. La compattezza tematica del canto si fonda in particolare sulla metafora della crescita e della fioritura spirituale che costituisce uno dei più significativi Leitmotive del poema ${ }^{24}$ : nel trionfo di Cristo i beati sono presentati come «[...] 'l frutto / ricolto del girar di queste spere!"» (vv. 20-21), il «[...] bel giardino / che sotto i raggi di Cristo s'infiora» (vv. 71-72) o ancora le «buone bobolce» ${ }^{25}$, che sono raccolto di una saggia seminagione. Al centro di questo sistema simbolico è la Vergine, a cui nel canto è dato rilievo nettamente maggioritario sul piano dello spazio narrativo: 27 versi - 19-45 - sono infatti destinati al trionfo di Cristo, 24 - vv. 46-69 - alla contemplazione della bellezza di Beatrice, ma ben 42 - vv. 70-111 - al trionfo di Maria. In particolare i vv. 73-74 («Quivi è la rosa in che 'l verbo divino / carne si fece [...]»), anticipa la preghiera di Bernardo in Par. XXXIII, 7-9: «Nel ventre tuo si raccese l'amore, / per lo cui caldo ne l'etterna pace / così è germinato questo fiore». Non solo ritroviamo qui un riferimento alla rosa mystica delle litanie che ci riporta al motivo dell'incarnazione di Cristo, ma anche alla funzione della Vergine come fondatrice e protettrice della Chiesa delle origini, come attesta l'esegesi medievale, ad esempio il Mariale aureum:

Rosa dicitur Virgo Maria, prout ipsa dicit: quasi plantatio Rosae in Jericho [Eccli 24:18]. Jericho interpretatur luna, et signat Ecclesiam militantem, in qua ad modum lunae, quamdiu est in praesenti vita multos habet defectus. In hac igitur Jericho, id est, in Ecclesia militanti ipsa est plantata, quia ipsa est plantata, quia ipsa inter nos et Deum est media constituta ${ }^{26}$.

17 Alla stessa costellazione metaforica appartiene anche un passo scritturale ben noto a Dante $^{27}$, quello dell'albero di Jesse (Is 11:1: «et egredietur virga de radice Jesse et flos de radice ejus ascendet») costantemente commentato dalla tradizione patristica di ambito mariano ${ }^{28}$, nonché tema di enorme fortuna in ambito artistico ${ }^{29}$. Il rilievo conferito dall'autore alla Vergine in Par. XXIII mi pare confermato dalla similitudine iniziale e da quella finale (vv. 1-9 e 121-123): l'«augello» - allodola o usignolo che sia ${ }^{30}$ - in attesa dell'alba ${ }^{31}$ per poter procacciare il cibo per i propri piccoli, e il neonato che tende le sue braccia alla madre dopo essere stato allattato ${ }^{32}$ corrispondono, in biunivoca specularità, a una figura di maternità amorevole e sollecita che non può che rimandare a Maria.

Dal momento, però, che la prima similitudine si riferisce esplicitamente a Beatrice, occorrerebbe a questo punto prendere posizione su una questione troppo vasta per essere affrontata in queste pagine, quella dei tratti mariani della "gentilissima» tra Vita 
nuova e Commedia. Nella Vita nuova l'intera parabola esistenziale di Beatrice sembra porsi sotto il segno della Vergine. Una delle prime epifanie di Beatrice avviene, com'è noto, «in parte ove s'udivano parole della Regina della gloria» $(2,6)$; la sua morte poi è così annunciata e commentata: «lo Signore della iustitia chiamòe questa gentilissima $a$ gloriare sotto la 'nsegna di quella Regina benedecta Maria, lo cui nome fue in grandissima reverenzia nelle parole di questa Beatrice beata» $(19,1)$. «Beata e bella» è Beatrice al suo primo apparire nelle parole di Virgilio (Inf. II, 53), quasi a confermare un'ascendenza mariana (Lc 1:48: «beatam me dicent omnes generationes»). Ancora alla fine della seconda cantica Dante sembra voler mantenere una sostanziale permeabilità tra le due figure, quando i ventiquattro seniori commentano l'apparizione di Beatrice intonando «Veni sponsa de Libano» (Ct 4:5; cfr. Purg. XXX, 11), versetto che l'esegesi medievale non solo intende come la Chiesa spirituale, ma anche come figura della Vergine ${ }^{33}$.

Nei canti finali del Paradiso assistiamo a un sostanziale cambiamento di strategia da parte dell'autore. Prima ancora che avvenga il «terremoto strutturale» che spezza la triade iniziale Maria - Lucia - Beatrice con l'assegnazione a Bernardo del compito di intermediario delle preghiere di Dante ${ }^{34}$, già Par. XXIII mette in scena una sorta di reindirizzamento dello sguardo del viator in favore della Vergine ad opera della stessa Beatrice:

«Perché la faccia mia sì t’innamora,

che tu non ti rivolgi al bel giardino

che sotto i raggi di Cristo s'infiora?

Quivi è la rosa in che 'l verbo divino carne si fece; $[. .]$.$» .$

(Par. XXIII, 70-74)

Vale la pena, a questo proposito, prendere in esame il passaggio dalla Vita nuova alla Commedia in una prospettiva particolare, ovvero quella che considera, per dir così, i mutamenti nella direzione degli sguardi. La scena della Vita nuova dell'incontro di Dante con Beatrice durante una funzione religiosa - probabilmente in Santa Maria Novella - cimostra una traiettoria lineare, da Dante a Beatrice, che solo accidentalmente (e provvidenzialmente) incontra sulla propria strada un'altra donna:

Uno giorno avenne che questa gentilissima sedea in parte ove s'udivano parole della Regina della gloria, e io era in luogo dal quale vedea la mia beatitudine; e nel mezzo di lei e di me per la recta linea sedea una gentil donna di molto piacevole aspecto, la quale mi mirava spesse volte, maravigliandosi del mio sguardare, che parea che sopra lei terminasse. Onde molti s'accorsero del suo mirare, e in tanto vi fue posto mente, che partendomi da questo luogo, mi sentio dire appressoi me: «Vedi come cotale donna distrugge la persona di costui»; e nominandola, intesi che dicea di colei che mezzo era stata nella linea recta che movea dalla gentilissima Beatrice e terminava negli occhi miei. Allora mi confortai molto, assicurandomi che lo mio secreto non era comunicato lo giorno altrui per mia vista. E immantanente pensai di fare di questa gentil donna schermo de la veritade, e tanto ne mostrai in poco di tempo, che lo mio secreto fu creduto sapere dalle più persone che di me ragionavano ${ }^{35}$.

21 Non appena Dante raggiunge il paradiso questa linearità si rifrange in duplicità, espressa dalla similitudine ottica del raggio incidente e di quello riflesso. Essa rettifica la scena precedente facendo di Beatrice non il destinatario finale dello sguardo dantesco, un Dio per ora lux inaccessibilis, ma un suo tramite: è lei ora a porsi come termine intermedio, non schermo ma specchio: 
Fatto avea di là mane e di qua sera

tal foce, e quasi tutto era là bianco

quello emisperio, e l'altra parte nera,

quando Beatrice in sul sinistro fianco

vidi rivolta e riguardar nel sole:

aquila sì non li s'affisse unquanco.

E sì come secondo raggio suole

uscir del primo e risalire in suso,

pur come pelegrin che tornar vuole,

così de l'atto suo, per li occhi infuso

ne l'imagine mia, il mio si fece,

e fissi li occhi al sole oltre nostr'uso.

[...]

Beatrice tutta ne l'etterne rote

fissa con li occhi stava; e io in lei

le luci fissi, di là sù rimote.

(Par. I, 43-54 e 64-66) che crede essere ancora la sua guida, e trova invece un misterioso «sene» che si rivelerà poi Bernardo di Chiaravalle (vv. 55 sgg.). È lui ad additare al viator Beatrice, già assisa in trono nella rosa dei beati: la distanza tra loro è ora immensa ${ }^{36}$, ma l'immagine si staglia netta perché non offuscata da alcuna impurità terrena (vv. 77-78: «[...] ché sua effige / non discendea a me per mezzo mista»). La linearità dello sguardo della Vita nuova parrebbe recuperata in una dimensione priva di ostacoli e accidenti, ma il nuovo destinatario della visione dantesca sarà in realtà ancora la Vergine:

«Riguarda omai ne la faccia che a Cristo

più si somiglia, ché la sua chiarezza

sola ti può disporre a veder Cristo» ${ }^{37}$.

(Par. XXXII, 85-87)

Beatrice esce a questo punto definitivamente dalla traiettoria dello sguardo di Dante. L'ultima sua apparizione non ha più carattere individuale, ma si confonde tra l'infinita schiera di beati che «chiudon le mani» in preghiera per Dante (Par. XXXIII, 38-39). Ora Maria si mostra con attributi pienamente regali - o addirittura imperiali: è «Agusta», Par. XXXII, $119^{38}-$, all'interno di una raffigurazione dal sapore cortese e cavalleresco di cui è emblema la «baldezza e leggiadria» dell'arcangelo Gabriele che, in un ennesimo richiamo all'Annunciazione, le porta la palma della vittoria.

Siamo giunti all'ultima tappa di questo breve excursus, la preghiera di Bernardo alla Vergine. Parto da un rilievo generale, ben conscio del suo valore relativo, a fronte della vastità della bibliografia dedicata al canto. Mi pare che la ricerca strenua all'individuazione, nell'opera di Bernardo in primo luogo, poi nella restante tradizione patristica, in quella liturgica e innografica, ecc., di singole suggestioni intertestuali per la preghiera di Par. XXXIII si sia rivelata sostanzialmente fallimentare ${ }^{39}$. I motivi sono evidenti: prima di tutto perché la tradizione mariana a cui essa attinge è talmente pervasiva e contaminata che appare impresa disperata poter determinare derivazioni certe; secondariamente perché la strategia elocutiva di Dante non è certo quella di un centonatore, ma di un autore geniale capace di rivitalizzare dall'interno i testi a cui si richiama, e di porli in relazione in un sistema complesso che ne rinnova i singoli significati. Lo schema canonico individuato da Auerbach ${ }^{40}$ sulla base degli studi di Norden, che permette di suddividere la preghiera di Bernardo in invocazione (vv. 1-3); elogio (vv. 4-21) e petizione (vv. 22-39) non comporta alcuna rigidità formulare: il testo 
infatti appare movimentato da immagini poetiche di straordinaria vitalità che generano una sorta di «declarative and consciously poetic theology»" nonché da quella dialettica tra discorso mistico apofatico, paradossale e fondato sulla dissomiglianza, e catafatico, fondato sulla rassegna delle virtutes di Maria, il cui modello appare Dionigi Areopagita $^{42}$. In effetti le coppie dei vv.1-2 Vergine-Madre, figlia-figlio, umile-alta non rappresentano delle semplici antitesi, ma polarità che opportunamente Georges Güntert ${ }^{43}$ ha definito «figure della totalità», le uniche adeguate per la Vergine, capace di conciliare gli opposti, avendo mostrato la sua centralità sia nella storia, di cui rappresenta il fondamentale Wendepunkt, sia nella dimensione eterna in cui è «meridiana face» ${ }^{44}$. Questa plenitudine amorevole di Maria, perfettamente còlta già nella poesia mediolatina, viene esaltata dalla particolare concentrazione semantica delle sintesi dantesche, che si avvale anche dell'ambiguità propria del discorso poetico: il «fiore» «germinato» dal ventre della Vergine, ad esempio, è contemporaneamente la rosa dei beati e Cristo, che ne è all'origine. Anche in questo caso il fondamento di questa ricchezza polisemica è riconducibile - ma senza risolversi completamente in essa - in una topica assai diffusa: oltre alla citata tradizione della Virga Jesse (Is 11:1) ${ }^{45}$, può essere richiamata l'antifona del tempo d'Avvento Rorate caeli desuper, sempre tratta dal libro di Isaia (45:8). Nella formula del responsorio dei Vespri, infatti, l'antifona presenta il versetto finale "Aperiatur terra et germinet Salvatorem», a differenza del «germinat salvationem» del passo biblico. Nel ricorso a formule di reciprocità come quelle delle prime due terzine di Par. XXXIII si può poi riconoscere qualche precedente nei testi latini del Medievo: ad esempio «nata nati» ${ }^{46}$ per «figlia del tuo figlio»; «cum tanta sublimitate humilitas» per "umile e alta» ${ }^{47}$; «factor fit ex factura» per la coppia fattore-fattura ${ }^{48}$.

Al di là della presunta influenza di questi spunti isolati, credo tuttavia che il passaggio esegetico cruciale sia quello dell'individuazione di testi che presentino una costellazione organica di riferimenti accostabili a quelli danteschi. Qualche tempo fa ho avuto modo di segnalarne uno la cui influenza appare ormai certa, dal momento che non solo entra in relazione con molteplici luoghi del poema, ma rivela una struttura generale assai affine a quella della Commedia, nel suo presentarsi come viaggio cosmologico di conoscenza e rigenerazione sul fondamento di una teologia poetica di ispirazione neoplatonica: mi riferisco all'Anticlaudianus di Alain de Lille ${ }^{49}$. Ecco dunque - e ciò valga come conclusione - il passo del libro V in cui Saggezza giunge sulla soglia del cielo delle Stelle fisse: per proseguire verso l'Empireo avrà bisogno dell'aiuto di Teologia, potendo così contemplare i cori angelici, il trionfo di Cristo e quello della Vergine ${ }^{50}$ :

Virgo que proprium pariendi lege pudorem Non perdens, matris meruit cum uirgine nomen ${ }^{51}$,

[...]

Hec est que miro diuini muneris usu

Nata patrem natumque parens concepit ${ }^{52}$, honorem

Virgineum retinens nec perdens iura parentis, In cuius uentris thalamo sibi summa parauit Hospicium deitas ${ }^{53}$, tunicam sibi texuit ipse Filius artificis summi, nostreque salutis Induit ipse togam, nostro uestibus amictu. Hec est stella maris, uite uia, porta salutis, Regula iusticie, limes pietatis ${ }^{54}$, origo Virtutis, uenie mater thalamusque pudoris, Ortus conclusus, fons consignatus ${ }^{55}$, oliua 
Fructiferans, cedrus redolens, paradisus amenans,

[...]

Spes miseris ${ }^{56}$, medicina reis, tutela beatis,

Proscriptis reditus, erranti semita, cecis

Lumen, deiectis requies, pausacio fessis.

[...]

Sic liber seruit ut seruos liberet, imum

Summa petunt ut sic ascendant infima summum ${ }^{57}$.

(Alain de Lille, Anticlaudianus V, 472-498 e 532-533)

La corrispondenza quasi perfetta nella successione dei parallelismi mostra, al di là delle vistose differenze stilistiche ${ }^{58}$, quanto opportuno fosse l'appello di Curtius ${ }^{59}$ a prendere in considerazione le opere della tradizione mediolatina tra i modelli più influenti della Commedia, la quale però tutti supera per altezza d'ingegno.

\section{NOTE}

1. Cfr. S. Botterill, Dante and the Mystical Tradition. Bernard of Clairvaux in the Commedia, Cambridge, Cambridge University Press, 1994, p. 154.

2. Sull'episodio di Inf. II, cfr. almeno A. M. Chiavacci Leonardi, Le tre donne benedette (Inferno II), in P. Sabbatino (a cura di), Realtà e simbolo della «donna» nella 'Commedia'. Lectura Dantis Pompeiana, Pompei, Biblioteca «L. Pepe», 1987, pp. 61-83.

3. Si discosta parzialmente dalla casistica «nostro Segnore» (Inf. XIX, 91).

4. Vita nuova 23, 7-8 (cito dall'ed. a cura di Guglielmo Gorni, Torino, Einaudi, 1996, pp. 193 sgg.); cfr. L. Ulrich, Das Sonett mit zwei Anfängen (Vita Nuova c. XXXIV), «Zeitschrift für Romanische Philologie», vol. LXX, 1954, pp. 376-388; M. Ciccuto, «Era venuta ne la mente mia» (V.N., XXXIV, 7): la visione nel libello e l'immagine di Dante, in V. Moleta (a cura di), "La gloriosa donna de la mente": A Commentary on the Vita nuova, Firenze-Perth, Olschki-Department of Italian, 1994, pp. 181-193, poi, col titolo «Era venuta ne la mente mia»: Visione nella Vita nuova e immagine nel pensiero di Dante, in Id., Icone della parola: Immagine e scrittura nella letteratura delle origini, Modena, Mucchi, 1995, pp. 95-112.

5. A. M. Chiavacci Leonardi, «In te misericordia, in te pietate». Maria nella Divina Commedia, in C. M. Piastra (a cura di), Gli studi di mariologia medievale. Bilancio storiografico, Atti del Convegno Mariologico della Fondazione Ezio Franceschini (Parma, 7-8 novembre 1997), Firenze, SISMEL Ed. del Galluzzo, 2001, pp. 321-334; cfr. p. 322.

6. Per un'analisi formale, cfr. L. Facini, Sì alta amanza à pres'a lo me' core, in A. Afribo, S. Bozzola e A. Soldani (a cura di), Le occasioni del testo. Venti letture per Pier Vincenzo Mengaldo, Padova, Univ. di Padova, 2016, pp. 9-26.

7. Com'è noto, nella Vita nuova il protagonista esprime sin dall'inizio del suo amore «una volontà di volere ricordare lo nome di quella gentilissima e acompagnarlo di molti nomi di donne» $(2,10$, ed. cit., p. 32).

8. Chiavacci Leonardi, «In te misericordia, in te pietate», cit., pp. 324 sgg.; B. Martinelli, Dante e il nome di Maria, in E. Ardissino (a cura di), Etica e teologia nella Commedia di Dante, Atti del seminario internazionale (Torino, 5-6 ottobre 2006), Roma, Ed. di Storia e Letteratura, 2009, pp. 85-114; cfr. p. 92. 
9. E. Bianchi, Maria, terra del cielo, introduzione a Maria: testi teologici e spirituali dal I al XX secolo, a cura della Comunità di Bose, Milano, A. Mondadori, 2000, p. XIII.

10. Par. XXV, 118-129: "Qual è colui ch'adocchia e s'argomenta / di vedere eclissar lo sole un poco, / che, per veder, non vedente diventa;// tal mi fec'io a quell'ultimo foco / mentre che detto fu: «Perché t'abbagli / per veder cosa che qui non ha loco? // In terra è terra il mio corpo, e saragli / tanto con li altri, che 'l numero nostro / con l'etterno proposito s'agguagli. // Con le due stole nel beato chiostro / son le due luci sole che saliro; / e questo apporterai nel mondo vostro». 11. P. J. Toynbee, Dante and the Legend of Saint John the Evangelist ("Par.", XXV, 100-102; 112-124), in Id., Dante Studies, Oxford, Clarendon Press, 1921, pp. 92-95; R. Jakoff, Dante and the Legend(s) of Saint John, «Dante Studies», $\mathrm{n}^{\circ}$ 117, 1999, pp. 45-57.

12. Mi permetto di rimandare a Canto XXV. «Ritornerò poeta», in E. Malato e A. Mazzucchi (a cura di), Lectura Dantis Romana. Cento canti per cento anni. III. Paradiso, 2. Canti XVIII-XXXIII, Roma, Salerno Ed., 2015, pp. 723-746; cfr. pp. 743 sgg.

13. Ancora Chiavacci Leonardi, «In te misericordia, in te pietate», cit., p. 325.

14. Corrado di Sassonia, Speculum Beatae Mariae Virginis, Lectio XV: Quod Maria sit benedicta per septem virtutes contra septem capitalia vitia, cito dall'ed. Firenze, Ad Claras Aquas, 1904, pp. 220 sgg. 15. Alcuni studiosi richiamano l'episodio dell'Annunciazione anche nella figura della «facella» che intona la melodia destinata alla Vergine di Par. XXIII, 94 sgg., identificata nell'arcangelo Gabriele; cfr. in particolare i vv. 103-105: «Io sono amore angelico, che giro / l'alta letizia che spira del ventre / che fu albergo del nostro disiro» (cfr. Luc 1:31: «concipies in utero et paries filium»). Per albergo, cfr. Conv. IV, v, 5: «E però [che] anche l'albergo dove il celestiale rege intrare dovea, convenia essere mondissimo e purissimo, ordinata fu una progenie santissima, de la quale dopo molti meriti nascesse una femmina ottima di tutte l'altre, la quale fosse camera del Figliuolo di Dio».

16. Cfr. Botterill, Dante and the Mystical Tradition, cit., pp. 157 sgg.

17. Botterill, Dante and the Mystical Tradition, cit., p. 159.

18. «Pia» sarà Beatrice testimoniando l'eccellenza dell'adesione alla speranza del proprio protetto quale esponente della «Chiesa militante»: «E quella pia che guidò le penne / de le mie ali a così alto volo, / a la risposta così mi prevenne: // "La Chiesa militante alcun figliuolo / non ha con più speranza, com'è scritto / nel Sol che raggia tutto nostro stuolo» (Par. XXV, 49-54). Per la Vergine si ricordi la conclusione dell'antifona Save regina (cfr. Purg. VII, 82): «O clemens, o pia, / o dulcis Virgo Maria». Forse non casualmente, anche Bernardo, nella sua prima, sorprendente apparizione, è descritto «in atto pio», con un riferimento all'atteggiamento paterno che richiama anche la prima guida: «Uno intendea, e altro mi rispuose: / credea veder Beatrice e vidi un sene / vestito con le genti gloriose. // Diffuso era per li occhi e per le gene / di benigna letizia, in atto pio / quale a tenero padre si convene» (Par. XXXI, 58-64 e cfr. Par. XXXII, 100: «santo padre»). Nove volte Virgilio è chiamato «dolce padre» nella Commedia (Inf. VIII, 110; Purg. IV, 44; XV, 25 e 124; XVII, 82; XVIII, 13; XXIII, 13; XXV, 17; XXVII, 52), e una volta semplicemente «padre» (Purg. XIII, 34).

19. Par. XIII, 84: «così fu fatta la Vergine pregna»; XVI, 34-36: «[...] "Da quel dì che fu detto 'Ave' / al parto in che mia madre, ch'è or santa, / s'alleviò di me ond'era grave» (sempre riferito a Cacciaguida); XXIII, 73-74: «Quivi è la rosa in che 'l verbo divino / carne si fece [...]»; XXIII, 104-105: «l'alta letizia che spira del ventre / che fu albergo del nostro disiro»; riferimenti che culminano in Par. XXXIII, 7-9: «Nel ventre tuo si raccese l'amore, / per lo cui caldo ne l'etterna pace / Così è geminato questo fiore».

20. La scuola francescana e l'Immacolata Concezione, Atti del Congresso mariologico francescano, S. Maria degli Angeli (Assisi, 4-8 dicembre 2003), Città del Vaticano, Pontificia Academia Mariana Internationalis, 2005.

21. R. Stefanini, Le tre mariofanie del Paradiso: XXII.88-129; XXXI.115-142; XXXII.85-114, «Italica», vol. 68, n 3, 1991, pp. 297-309. 
22. M. Perugi, Canto XXIII, in G. Güntert e M. Picone (a cura di), Lectura Dantis Turicensis, Firenze, Cesati, 2000-2002, III, pp. 363-371; cfr. p. 364.

23. Ivi, pp. 364-370.

24. Cfr. il mio Il diletto legno. Aridità e fioritura spirituale nella Commedia, Firenze, Olschki, 1994.

25. Mi pare che, in virtù di questo parallelismo, sia da preferire l'accezione di "campo" (tardo lat. bubulca) su quello di "lavoratori della terra", che rimanderebbe agli apostoli.

26. Mariale aureum, a cura di Adel Figarol, Tolosa, Ex Typis Orphanarum Sancti Josephi Boni Auxilii, 1874, XVII, 4, p. 353. Com'è noto, anche la luna (cfr. «Trivïa» in Par. XXIII, 26) rientra nella simbologia mariana.

27. Conv. IV, v, 3-6: «Volendo la 'nmensurabile bontà divina l'umana creatura a sé riconformare, che per lo peccato de la prevaricazione del primo uomo da Dio era partita e disformata, eletto fu in quello altissimo e congiuntissimo consistorio de la Trinitade, che 'l Figliuolo di Dio in terra discendesse a fare questa concordia. [...] E però [che] anche l'albergo dove il celestiale rege intrare dovea, convenia essere mondissimo e purissimo, ordinata fu una progenie santissima, de la quale dopo molti meriti nascesse una femmina ottima di tutte l'altre, la quale fosse camera del Figliuolo di Dio: e questa progenie fu quella di David, del qual [di]scese la baldezza e l'onore de l'umana generazione, cioè Maria. E però è scritto in Isaia: "Nascerà virga de la radice di Iesse, e fiore de la sua radice salirà"».

28. Ad es. Bernardo di Chiaravalle, Sermones de tempore, Homiliae quatuor super Missus est, II, 5 , in PL 185, 63: «Huius magni miraculi [scil.: del bastone di Aronne] maius mysterium Isaias edisserit, dicens "Egredietur virga de radice Jesse, et flos de radice eius ascendet": virgam Virginem, florem Virginis partum intelligens».

29. Cfr. A. Watson, The Early Iconography of the Tree of Jesse, London, Milford, 1934, che ricorda il pannello del pulpito di S. Leonardo in Arcetri a Firenze (fine sec. XII), in cui la Vergine viene effigiata con il Bambino. Cfr. anche, dello stesso autore, The Speculum Virginum with Special Reference to the Tree of Jesse, «Speculum», vol. 3, $\mathrm{n}^{\circ} 4,1928$, pp. 445-449. Sulle raffigurazioni dell'albero di Jesse sul portale del Duomo di Orvieto, cfr. P. Salonius, The Tree of Jesse, the Tree of Life, and the Mendicants in Late Medieval Orvieto, in P. Salonius e A. Worm (a cura di), The Tree. Symbol, Allegory, and Mnemonic Device in Medieval Art and Thought, Turnhout, Brepols, 2014, pp. 213-241.

30. Cfr. Perugi, Canto XXIII, cit., pp. 364 sgg.

31. Altro attributo mariano è quello di Stella matutina (Eccli 50:6), ricordato anche da Bernardo in Par. XXXII, 108; nelle litanie medievali troviamo anche quello di Aurora consurgens; cfr. G. G. Meersseman, „Virgo a doctoribus praetitulata“. Die marianischen Litaneien als dogmengeschichtliche Quellen, «Freiburger Zeitschrift für Philosophie und Theologie», vol.1, 1954, pp.129-178; cfr. p. 166.

32. Immagine che l'autore riserva a se stesso nel momento decisivo della visione finale: cfr. Par. XXXIII, 106-108: «Omai sarà più corta mia favella, / pur a quel ch'io ricordo, che d'un fante / che bagni ancor la lingua a la mammella».

33. Ad esempio in Ugo di San Vittore: cfr. L. Pertile, La puttana e il gigante. Dal «Cantico dei cantici» al Paradiso Terrestre di Dante, Ravenna, Longo, 1998, pp. 62 sgg.

34. Id., La punta del disio. Semantica del desiderio nella Commedia, Firenze, Cadmo, 2005, p. 245; ma vedi tutto il cap. IX Dimenticare Beatrice. La tesi di fondo del saggio è che nell'epilogo del poema l'amore per Dio finisce per sostituire e dissolvere quello per Beatrice.

35. Vita nuova 2, 6-8, ed. cit., pp. 29-30.

36. Par. XXXI, 73-76: «Da quella region che più sù tona / occhio mortale alcun tanto non dista, / qualunque in mare più giù s'abbandona, // quanto lì da Beatrice la mia vista».

37. Sulle corrispondenze numerologiche e geometriche della triplice rima "Cristo", cfr. T. E. Hart, The Cristo-Rhymes and Polyvalence as a Principle of Structure in Dante's Commedia, "Dante Studies», $\mathrm{n}^{\circ}$ 105, 1987, pp. 1-42; Id., The Cristo-Rhymes, the Greek Cross, and Cruciform Geometry in Dante's 
Commedia: "giunture di quadranti in tondo», «Zeitschrift für romanische Philologie», vol. 106, $\mathrm{n}^{\circ} 1-2,1990$, pp. 106-134.

38. La Vergine era stata chiamata «regina» in Par. XXXI, 116 e XXXII, 104.

39. Vedi soprattutto C. Cavedoni, Raffronti tra gli autori biblici e sacri e la Divina Commedia, Lapi, Citta di Castello, 1896 e A. Vallone, Studi su Dante medievale, Firenze, Olschki, 1965, pp. 86 sgg.

40. E. Auerbach, La preghiera di Dante alla Vergine (Par. XXXIII) ed antecedenti elogi, in Id., Studi su Dante [1949], Milano, Feltrinelli, 1971, pp. 263-292.

41. Botterill, Dante and the Mystical Tradition, cit., p. 164.

42. M. Ariani, La mistica preterizione: il "dicer poco" dell'ultimus cantus, in E. Malato e A. Mazzucchi (a cura di), Lectura Dantis Romana. Cento canti per cento anni. III. Paradiso, 2. Canti XVIII-XXXIII, Roma, Salerno Ed., 2015, pp. 971-1011. Sull'importanza decisiva di Dionigi per la conclusione del poema, cfr. il mio Dante e lo Pseudo-Dionigi: una nuova proposta per l'immagine finale della Commedia, «Lettere italiane», vol. 61, nº 1, 2009, pp. 3-29.

43. G. Güntert, Canto XXXIII, in Lectura Dantis Turicensis, cit., III, pp. 505-518; cfr. p. 514.

44. Sulle scelte formali di questi versi, cfr. anche A. Pennacini, Retorica e teologia nel canto XXXIII del 'Paradiso', in G. Beccaria e C. Marello (a cura di), La parola al testo. Scritti per Bice Mortara Garavelli, Alessandria, Edizioni dell'Orso, 2002, II, pp. 933-942.

45. Vedi ad es. Bernardo di Chiaravalle, Sermones de tempore, Homiliae quatuor super Missus est, II, 6, in PL 185, 64: «In hoc tamen Isaiae testimonio, florem Filium, virgam intellige Matrem; quaniam et virga floruit absque germine, et virgo concepit non ex homine. Nec virgae virorem floris laesit emissio, nec virginis pudorem sacri partus editio».

46. Analecta Hymnica Medii Aevi, a cura di G. M. Dreves e C. Blume [1886-1922], vol. VIII, 89, De beata Maria, p. 73: «Verbum Patris concepisti / Patrem nata peperisti / mundo ferens gaudia // Nata Nati, mater Patris, / Modo miro nomen matris / habes viri nescia».

47. Bernardo di Chiaravalle, Sermones de tempore, cit., I, 9, in PL 185, 61: «Mirum vero si non haesitas in eorum ponderatione, quid tua iudices dignius admiratione, utrum videlicet potius stupenda sit fecunditas in virgine, an in matre integritas; sublimitas in prole, an cum tanta sublimitate humilitas»?

48. Pier Damiani, Sermo XLV In nativitate beatissimae virginis mariae, in PL 144, 742-743: «Cum vero beatissimae Genitricis Dei laudes scribere volumus, quia nova et inaudita sunt quae tractanda suscipimus, nulla invenimus verba quibus ad haec digne exprimenda sufficere valeamus. Tollit enim facultatem sermonis materia singularis. Quae enim lingua explicare sufficiat, vel quae ratio humana non obstupescat, cum cogitare coeperit quia Creator oritur ex creatura, factor fit ex factura? Quod in virginea puellae vulva concipitur, qui vastissima totius mundi amplitudine non tenetur? Iacet intra materna viscera parvulus, qui cum coaeterno Patre rerum omnium jura gubernat immensus»?

49. Cfr. i miei Teologia come pittura: Alain de Lille e Dante (Purg. XI, 82-84), in M. Ariani, A. Bruni e A. Dolfi, A. Gareffi (a cura di), La parola e l'immagine. Studi in onore di Gianni Venturi, Firenze, Olschki, 2011, pp. 99-116 e «Ad intuitum supercelestium formarum»: Alain de Lille e la Commedia, in G. Ledda (a cura di), Dante e la cultura religiosa medievale. In ricordo di Anna Maria Chiavacci Leonardi, Atti del Convegno internazionale di studi (Ravenna, 26 novembre 2015), Ravenna, Centro Dantesco dei Frati Minori Conventuali, 2018, pp. 117-136.

50. Cito dall'ed. a cura di Carlo Chiurco, Milano, Bompiani, 2004, pp. 222-224.

51. XXXIII, 1: «Vergine madre».

52. XXXIII, 1: «figlia del tuo figlio».

53. XXXIII, 7: «Nel ventre tuo si raccese l'amore».

54. XXXIII, 19-20: «in te pietate / [...] s'aduna».

55. XXXIII, 12: «fontana vivace».

56. Ancora XXXIII, 12: «se' di speranza fontana vivace».

57. XXXIII, 22-23: «Or questi, che da l'infima lacuna / de l'universo infin qui ha vedute». 
58. Se, infatti, Alain de Lille ricorre a una complessa ipotassi di inesauribile ricchezza metaforica, Dante si affida al contrario, in particolare nella prima terzina, a «uno stile nominale di puri contrapposti binari» (cfr. Ariani, La mistica preterizione, cit., pp. 973 sgg.).

59. E. R. Curtius, Europäische Literatur und lateinisches Mittelalter [1948], trad. ital. Letteratura europea e Medio Evo latino, cito dall'edizione a cura di R. Antonelli, Firenze, La Nuova Italia, 1993, p. 390.

\section{RIASSUNTI}

La rappresentazione letteraria della figura di Maria costituisce un tema particolarmente insidioso in un'opera di argomento religioso come la Commedia, sia per le controversie dogmatiche che al tempo di Dante la riguardavano, come l'immacolata concezione, sia per l'effetto di ridondanza causato dall'enorme sviluppo della tradizione mariana, dalle leggende alla liturgia all'innografia. Dante affronta queste difficoltà da un lato presentando una selezione estremamente sobria degli episodi della vita di Maria, tratta rigorosamente dal solo testo evangelico; dall'altro rendendo estremamente dinamico il ritratto della sua figura, in particolare attraverso la progressione che si realizza tra la seconda e la terza cantica, dalla Vergine terrena alla Regina celeste. Se il Purgatorio pone l'aspetto della maternità come centrale, mettendo in particolare risalto il momento dell'Annunciazione, nel Paradiso Maria tende a concentrare in sé il potere salvifico che la poesia di Dante aveva affidato, attraverso Beatrice, alla presenza femminile. Negli ultimi canti del Paradiso, in particolare, la sostituzione di Beatrice da parte di Bernardo si accompagna alla perdita di centralità di quest'ultima, aspetto che il saggio analizza attraverso un'analisi comparativa di Vita nuova e Commedia. Il saggio si conclude indicando l'invocazione alla Vergine dell'Anticlaudianus di Alain de Lille quale intertesto esteso della preghiera finale di Bernardo.

La représentation littéraire de la figure de Marie constitue un thème particulièrement insidieux dans une œuvre à sujet religieux telle que la Divine Comédie, tant en raison des controverses dogmatiques qui la concernaient à l'époque de Dante, comme l'immaculée conception, que de l'effet de redondance provoqué par l'énorme développement de la tradition mariologique, des légendes à la liturgie et à l'hymnographie. Dante fait face à ces difficultés d'une part en présentant une sélection extrêmement restreinte d'épisodes de la vie de Marie, tirés du seul texte évangélique; d'autre part en faisant de sa figure un portrait extrêmement dynamique, notamment à travers la progression qui se déroule entre le deuxième et le troisième cantique, de la Vierge terrestre à la Reine céleste. Si le Purgatoire souligne la centralité du thème de la maternité, en insistant notamment sur le moment de l'Annonciation, Marie tend dans le Paradis à concentrer en elle le pouvoir salvateur que la poésie de Dante avait confié, à travers Béatrice, à la présence féminine. Dans les derniers chants du Paradis, en particulier, le remplacement de Béatrice par saint Bernard entraîne une perte de centralité de cette dernière : la présente étude met en évidence cet aspect à travers une analyse comparative de la Vita nuova et de la Divine Comédie. On reconnaît pour finir dans l'invocation à la Vierge de l'Anticlaudianus d'Alain de Lille l'intertexte de la prière finale de Bernard.

The literary representation of the figure of Mary is a particularly insidious theme in a religious work such as the Commedia, both because of the dogmatic controversies that concerned her at Dante's time, such as the immaculate conception, and because of the effect of redundancy caused 
by the enormous development of the Marian tradition, from legends to liturgy to hymnography. Dante faces these challenges on the one hand by presenting an extremely sober selection of episodes from Mary's life, taken strictly from the Gospel text alone; on the other by making the portrait of her figure extremely dynamic, particularly through the progression that takes place between the second and third canticles, from the earthly Virgin to the heavenly Queen. If the Purgatory places the aspect of motherhood as central, highlighting in particular the moment of the Annunciation, in Paradise Mary tends to concentrate in herself the saving power that Dante's poetry had entrusted, through Beatrice, to the female presence. In the last Cantos of Paradise, in particular, Bernard's replacement of Beatrice is followed by the latter's loss of centrality, an aspect that the essay analyses through a comparative analysis of Vita nuova and Commedia. The essay concludes by indicating the invocation to the Virgin in Alain de Lille's Anticlaudianus as the extended intertext of Bernard's final prayer.

\section{INDICE}

Mots-clés : tradition mariologique, Mariophanies, Septénaire des vertus, Alain de Lille

Parole chiave : tradizione mariana, Mariofanie, Settenario delle virtù, Alain di Lille

Keywords : Marian tradition, Mariophanies, Septenary of virtues, Alain of Lille

\section{AUTORE \\ STEFANO PRANDI}

Stefano Prandi si è formato all'Università di Bologna con Ezio Raimondi. Dal 2001 al 2017 è stato titolare della Cattedra di Letteratura italiana all'Università di Berna, e attualmente è Direttore dell'Istituto di studi italiani presso l'Università della Svizzera Italiana. Nell'ambito degli studi danteschi ha realizzato un'edizione integrale commentata della Commedia (Brescia, La Scuola, 2005), una monografia e numerosi saggi. Ha infine curato per l'editore Olschki «Nel lago del cor». Letture di Dante all'Università della Svizzera italiana (2012-2016).

Stefano Prandi s'est formé à l'Université de Bologne avec Ezio Raimondi. De 2001 à 2017 il a occupé la chaire de littérature italienne à l'Université de Berne, et est actuellement directeur de l'Institut d'études italiennes de l'Université de la Suisse italienne. Dans le domaine des études sur Dante, il a produit une édition complète annotée de la Divine Comédie (Brescia, La Scuola, 2005), une monographie et de nombreux essais. Enfin, il a édité pour Olschki «Nel lago del cor». Letture di Dante all'Università della Svizzera italiana (2012-2016). 